\title{
Reactive Hepatitis in a Case of Celiac Disease
}

\author{
Sur Genel ${ }^{1,2, *}$, Donca Valer ${ }^{3}$, Sur M Lucia ${ }^{1}$, Sur Daniel ${ }^{1}$, Floca Emanuela ${ }^{1}$, Samasca Gabriel ${ }^{1,2}$ \\ ${ }^{1}$ Iuliu Hatieganu University of Medicine and Pharmacy, Cluj-Napoca, Romania \\ ${ }^{2}$ Emergency Clinical Hospital for Children, Cluj-Napoca, Romania \\ ${ }^{3}$ Clujana Municipal Hospital, Cluj-Napoca, Romania \\ *Corresponding author: surgenel@yahoo.com
}

Received September 05, 2014; Revised September 20, 2014; Accepted October 10, 2014

\begin{abstract}
Liver injury in celiac disease may evolve from reactive hepatitis to autoimmune hepatitis. Our case is a reactive hepatitis evolves parallel with celiac disease activity. If the patient does not follow the gluten free diet both manifestations of celiac disease and reactive hepatitis will appear. Reactive hepatitis manifestations can be rectified after 6-12 months of gluten-free diet.
\end{abstract}

Keywords: liver, transaminases, gluten-free diet

Cite This Article: Sur Genel, Donca Valer, Sur M Lucia, Sur Daniel, Floca Emanuela, and Samasca Gabriel, "Reactive Hepatitis in a Case of Celiac Disease." International Journal of Celiac Disease, vol. 2, no. 4 (2014): 137-138. doi: 10.12691/ijcd-2-4-3.

\section{Introduction}

The primary manifestations of celiac disease are gastrointestinal symptoms. There are also described extraintestinal manifestations such as skin, heart, nervous system and the hepatobiliary system [1,2]. In untreated celiac disease elevation of serum transaminases (especially aminotransferase) levels is common in 15-55\% of patients. Celiac disease has been found in as many as $9 \%$ of patients with elevated aminotransferase. A probable mechanism is autoimmunity and genetic predisposition [3]. Another pathogenetic mechanism consists in increased permeability of intestinal mucosa, allowing absorption of antigens from the gut. These antigens could induce an immune response and exposure of tissue transglutaminase enzyme to the anti-endomysial antibody. This autoimmune antibody plays a major role in inducing liver injury [4]. Liver impairment in celiac disease includes mild hepatic parenchymal inflammation which is reversible after gluten-free diet, chronic inflammation of the liver which is irreversible after gluten-free disease and potentially reversible liver failure after gluten-free diet [5]. Other possible liver injuries in celiac disease are fatty liver, nodular regeneration, cryptogenic cirrhosis and liver hyperplasia. Reactive hepatitis is common as a manifestation in celiac disease. Elevated liver enzymes are mild to moderate and liver function is remedied after 6-12 months of $100 \%$ gluten-free diet [6].

\section{Case Report}

We report the case of a girl who at the age of 1 year and 5 months was hospitalized for numerous stools in large quantity and weight loss.
She was born full term, following a normal pregnancy; birth weight was $2800 \mathrm{~g}$, birth score 9 and she was exclusively breast fed to 6 months. In family history there is no significant pathology mentioned.

After the beginning food diversification parents notice the appearance of accelerated bowel transit with numerous modified stools. Over time these symptoms has influenced the growth rate. Parents noticed weight stagnation followed by weight loss lately.

Clinical examination revealed pale and dry skin. Subcutaneous tissue was poorly represented generalized patient weighing $8 \mathrm{~kg}$ (value below the 5 th percentile on the growth chart for age). No pathological changes were detected in the respiratory and cardio-circulatory system. It was also revealed abdominal distension, gracile limbs and hypotonic.

Laboratory examinations showed that antitransglutaminase-IgA and anti-endomysium-IgA were positive. The child presented a syndrome of hepatocitolyse with a slight increase of serum transaminases. In these conditions we investigated a possible infectious hepatitis. We determined HBs antigen and HCV antibodies which were negative, thus excluding infection with hepatitis viruses $\mathrm{B}$ and $\mathrm{C}$. We excluded acute infection with cytomegalovirus (CMV IgM antibodies were negative), and Toxoplasma gondii (Toxoplasma IgM antibody negative).

Pathological examination of duodenal biopsy showed villous atrophy, Marsh type 3.

Thus we established the diagnosis of celiac disease and reactive hepatitis.

Therapeutic recommendation was gluten-free diet and treatment with hepatoprotectors. By gluten-free diet evolution was spectacular with progressive and constant increase in weight.

On clinical reassessment at age 10, laboratory examinations revealed pathological values of anti- 
transglutaminase-IgA and anti-endomisium-IgA. Serum transaminases were elevated and the hemoglobin to lower limit for the age. Abdominal ultrasound showed a liver with normal structure and dimensions. Although the patient tried to keep a gluten-free diet, the food, especially flour products, it seems that they contain traces of gluten. It is possible that patients may have consumed products with gluten in certain circumstances such as play meetings with friends, parties, birthdays. For this reason, the antitransglutaminase IgA antibodies were again positive and digestive manifestations reappeared with bulky stools, failure to thrive, and an incipient deficiency syndrome with mild anemia. Caution is advised in diet, and especially to the use of those dishes that is mentioned that do not contain gluten.

\section{Particularities of the Case}

A particular aspect of the case is the occurrence of hepatitis which I thought was reactive without an infectious cause. According to the literature it is possible the appearance of the reactive hepatitis to be maintained throughout the immune mechanism.
Despite a gluten-free diet the symptoms reappeared and anti-transglutaminase-IgA were positive because the products were not safe as $100 \%$ gluten-free. Therefore, patients on a gluten-free diet must carefully read the label on gluten-free products.

\section{References}

[1] Prashant Singh, Govind K Makharia. Non-classical Celiac Disease Often Missed. International Journal of Celiac Disease 2014; 2: 7685.

[2] Sur G, Floca E, Sur M Lucia, Sur G Daniel, Samasca G. Clinical Presentation of Celiac Disease Masks Therapeutic Perspectives of Celiac Disease. Pharmaceut Anal Acta 2013; 4: 1000228.

[3] Reilly NR, Fasano A, Green PH. Presentation of celiac disease. Gastrointest Endosc Clin N Am 2012; 22: 613-621.

[4] Di Sabatino A, Vanoli A, Giuffrida P, Luinetti O, Solcia E, Corazza GR. The function of tissue transglutaminase in celiac disease. Autoimmunity Reviews 2012; 11: 746-753.

[5] Drastich P, Honsová E, Lodererová A, Jarešová M, Pekáriková A, Hoffmanová I, Tučková L, Tlaskalová-Hogenová H, Spičák J, Sánchez D. Celiac disease markers in patients with liver diseases: A single center large scale screening study. World J Gastroenterol 2012; 18: 6255-6262.

[6] Rubio-Tapia A, Murray JA. The liver in celiac disease. Hepatology 2007; 46: 1650-1658. 